\title{
ANALYTICAL SOLUTION OF THE PROBLEM ON MHD FLOW IN DUCT WITH PERFECTLY CONDUCTING HARTMANN WALLS AND SLIP CONDITION ON CONDUCTING SIDE WALLS
}

\author{
Elena Ligere, Ilona Dzenite, Aleksandrs Matvejevs \\ Riga Technical University, Latvia \\ jelena.ligere@rtu.lv, ilona.dzenite@rtu.lv, aleksandrs.matvejevs@rtu.lv
}

\begin{abstract}
An analytical solution has been obtained for a problem on magnetohydrodynamic flow in a rectangular duct with perfectly conducting Hartmann walls and side walls of finite conductivity. The slip boundary conditions for velocity were applied to the duct side walls. The exact analytical solution of the problem has been obtained in the form of convergent series. On the base of the obtained analytical solution the velocity field of the flow has been studied numerically.
\end{abstract}

Keywords: MHD duct flow, slip boundary condition.

\section{Introduction}

A flow of electrically conducting fluid in the presence of external magnetic field produces a variety of new effects, which are not realizable in usual hydrodynamics. These effects are studied by magnetohydrodynamics (MHD), the discipline combining the classical fluid mechanics and electrodynamics.

Electromagnetic methods of action on electrically conducting medium are widely exploited in industrial processes in metallurgy, material processing, chemical industry, industrial power engineering and nuclear engineering in order to control and manipulate various conducting materials. Besides, the MHD effects are exploited in technical devices such as MHD pumps, flow meters, MHD generators and accelerators, where channels with electrically conducting fluids are their common parts. Thus, investigation of MHD phenomena in channels with conducting fluids is quite important.

In magnetohydrodynamics the number of exact analytical solutions is limited due to the nonlinearity of the Navier-Stokes equations. Therefore, numerical methods are widely used for solving these problems, although analytical solutions still do not lose their importance (even if these solutions are often obtained for simplified flows). The present analytical study was motivated by the recent publication [2], in which three classical MHD problems were considered, but the applied boundary condition at the interface between the electrically conducting fluid and the insulating solid walls was the hydrodynamic slip condition. Despite the no-slip boundary condition is usually applied for the majority of flows of viscous fluids, there exist situations when no-slip boundary conditions lead to unrealistic behaviour, for example, if viscous effects at the wall are negligible, then the slip boundary condition must be applied (see [1;2]).

This paper presents an exact analytical solution obtained for a new problem on a MHD flow in the rectangular duct with perfectly conducting Hartmann walls and the side walls of finite conductivity. The boundary condition applied to the Hartmann walls of the duct is the Dirichlet (no-slip) boundary condition, which is the simplest boundary condition for the velocity of viscous fluid, while the boundary condition applied to the side walls is the 3rd kind (slip) boundary condition. This solution seems absent in literature. Some numerical results for the velocity of the flow calculated on the base of the obtained analytical solution are also presented. It is to be noted that the study of the present problem continues, so that additional calculations and their interpretations are expected in the nearest future.

\section{Materials and methods}

A rectangular duct with incompressible, electrically conducting fluid is located in an imposed uniform transverse magnetic field $\vec{B}^{e}=B_{0} \vec{e}_{z}$. The Hartmann walls of the channel at $\tilde{z}= \pm b$ are perfectly conducting, but the side walls at $\tilde{y}= \pm a$ have finite conductivity. The flow in the duct is driven by a constant pressure gradient kept along the duct. The slip boundary conditions are given on the on the side walls (see Fig. 1). 


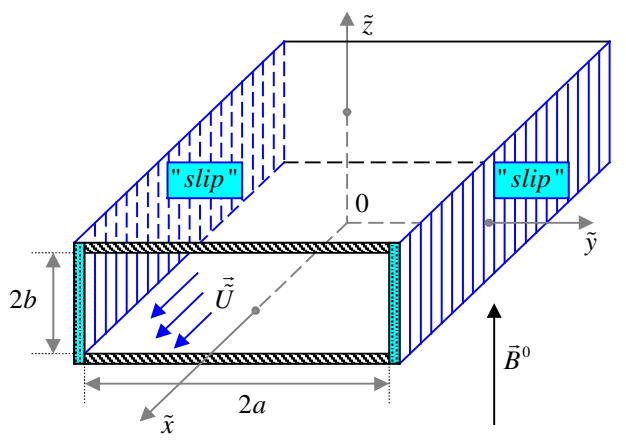

Fig. 1. MHD duct flow with a slip boundary condition

The problem is described by the following dimensionless MHD equations (see $[2 ; 3]$ ):

$$
\begin{gathered}
\frac{\partial^{2} U}{\partial z^{2}}+\frac{\partial^{2} U}{\partial y^{2}}+H a \frac{\partial b_{x}}{\partial z}+1=0, \\
\frac{\partial^{2} b_{x}}{\partial z^{2}}+\frac{\partial^{2} b_{x}}{\partial y^{2}}+H a \frac{\partial U}{\partial z}=0,
\end{gathered}
$$

where $\vec{V}=U(y, z) \cdot \vec{e}_{x}-$ the velocity of the fluid;

$\vec{b}=b_{x}(y, z) \cdot \vec{e}_{x}-$ the induced magnetic field;

$H a=B_{0} b \sqrt{\sigma / \rho v}$ - the Hartmann number;

$\sigma, \rho, v-$ the conductivity, the density and the viscosity of the fluid, respectively.

The dimensionless boundary conditions are

$$
\begin{gathered}
z= \pm 1: U=0, \frac{\partial b_{x}}{\partial z}=0, \\
y= \pm d: U \pm \alpha \cdot \frac{\partial U}{\partial y}=0, b_{x} \pm \gamma \cdot \frac{\partial b_{x}}{\partial y}=0,
\end{gathered}
$$

where $\alpha$-the slip length of fluid;

$\gamma$ - the coefficient of conductivity of the side walls;

$y=\tilde{y} / b, z=\tilde{z} / b, d=a / b$, the values $\tilde{x}, \tilde{y}, \tilde{z}$ are dimensional.

The problem is solved by using the Fourier cosine and sine expansions of the velocity and the induced magnetic field, with respect to $\mathrm{z}$ :

$$
\begin{aligned}
& U(y, z)=\sum_{n=0}^{\infty} \bar{u}\left(a_{n}, y\right) \cdot \cos \left(a_{n} z\right) \\
& b_{x}(y, z)=\sum_{n=0}^{\infty} \bar{b}\left(a_{n}, y\right) \cdot \sin \left(a_{n} z\right)
\end{aligned}
$$

where $a_{n}=\pi n+\pi / 2, n=0,1,2, \ldots$ is taken to satisfy boundary conditions (4).

The solution of the problem (1)-(4) is obtained in the form of the convergent series:

$$
U=\sum_{n=0}^{\infty} \frac{2(-1)^{n} i}{\left(a_{n}^{2}+H a^{2}\right) a_{n}^{2}}\left(\frac{\tilde{C}_{n} \cosh \left(k_{1} y\right)-\tilde{D}_{n} \cosh \left(k_{2} y\right)}{\Delta}-i a_{n}\right) \cos \left(a_{n} z\right)
$$




$$
b_{x}=\sum_{n=0}^{\infty} \frac{2(-1)^{n}}{\left(a_{n}^{2}+H a^{2}\right) a_{n}^{2}}\left(\frac{\tilde{C}_{n} \cosh \left(k_{1} y\right)+\tilde{D}_{n} \cosh \left(k_{2} y\right)}{\Delta}-H a\right) \sin \left(a_{n} z\right)
$$

where

$$
\begin{gathered}
k_{1}=\sqrt{a_{n}^{2}+i a_{n} H a}, k_{2}=\sqrt{a_{n}^{2}-i a_{n} H a}, \\
\tilde{C}_{n}=\left(H a+i a_{n}\right) \cosh \left(k_{2} d\right)+\left(\alpha H a+i \gamma a_{n}\right) \cdot k_{2} \sinh \left(k_{2} d\right) \\
\tilde{D}_{n}=\left(H a-i a_{n}\right) \cosh \left(k_{1} d\right)+\left(\alpha H a-i \gamma a_{n}\right) \cdot k_{1} \sinh \left(k_{1} d\right) \\
\Delta=2 \cosh \left(k_{1} d\right) \cosh \left(k_{2} d\right)+(\alpha+\gamma)\left(k_{1} \sinh \left(k_{1} d\right) \cosh \left(k_{2} d\right)+k_{2} \cosh \left(k_{1} d\right) \sinh \left(k_{2} d\right)\right)+ \\
+2 \alpha \gamma k_{1} k_{2} \sinh \left(k_{1} d\right) \sinh \left(k_{2} d\right)
\end{gathered}
$$

\section{Results and discussion}

On the base of the obtained analytical solution the velocity of the flow was studied numerically. In Fig. 2 the velocity profiles $U / U_{m}$ are presented for different $\alpha$ and $\gamma$ values at $H a=100$, where $U_{m}$ is the mean bulk velocity of the flow.
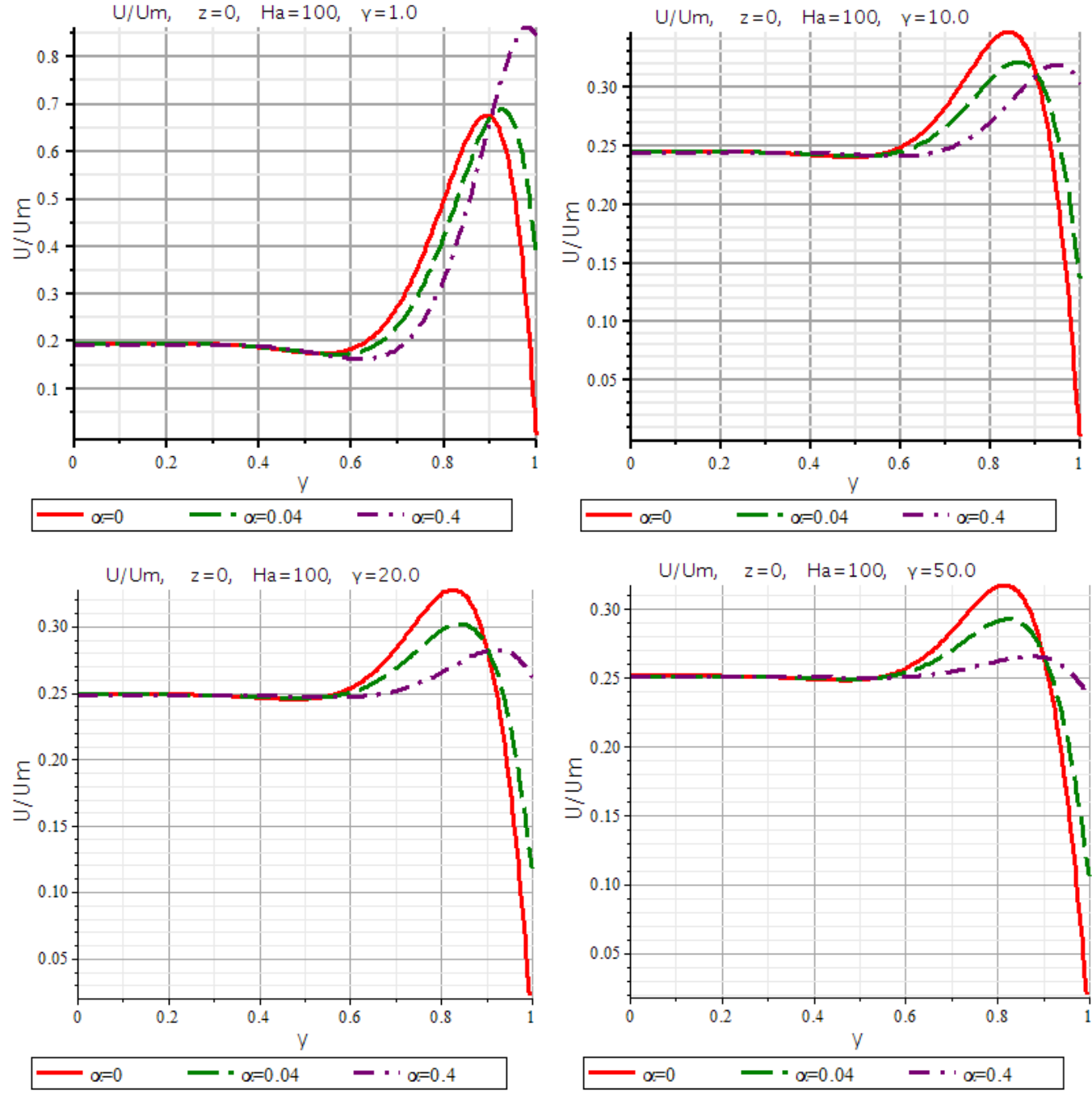

Fig. 2. Velocity profiles for different $\alpha$ and $\gamma$ values at $H a=100$ 
$\alpha=0.04, \quad \gamma=5, \quad \mathrm{Ha}=10$
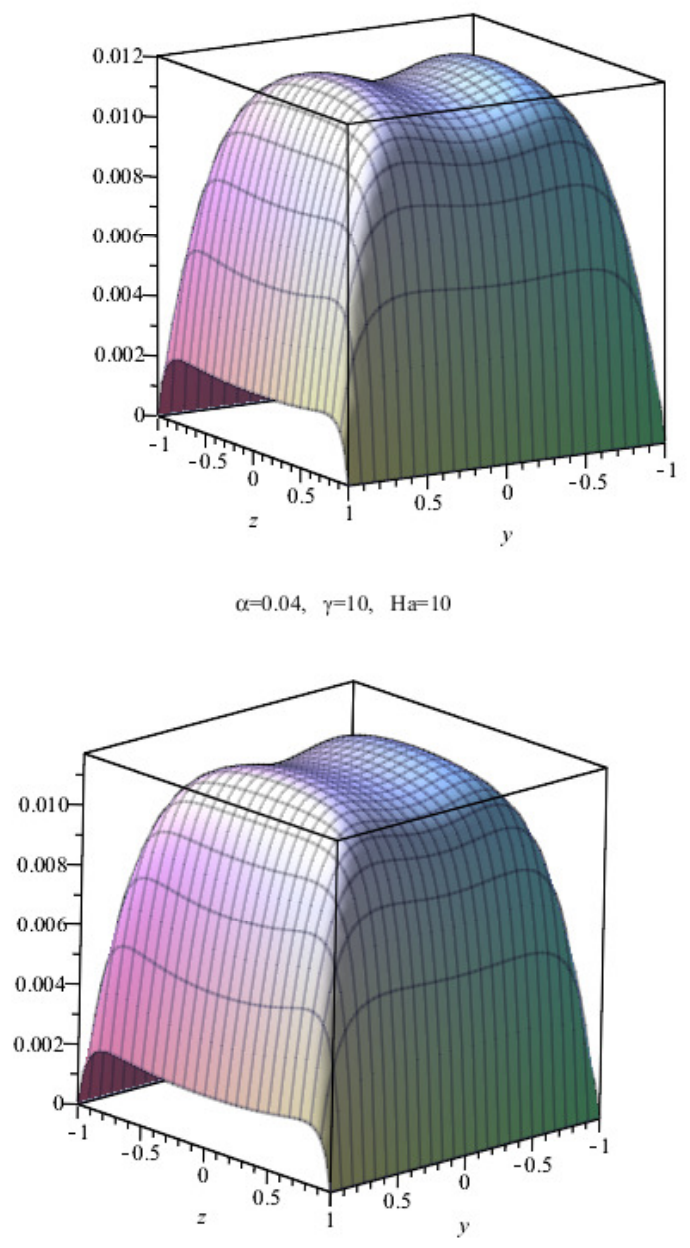

$\alpha=0.04, \quad \gamma=10, \quad \mathrm{Ha}=100$

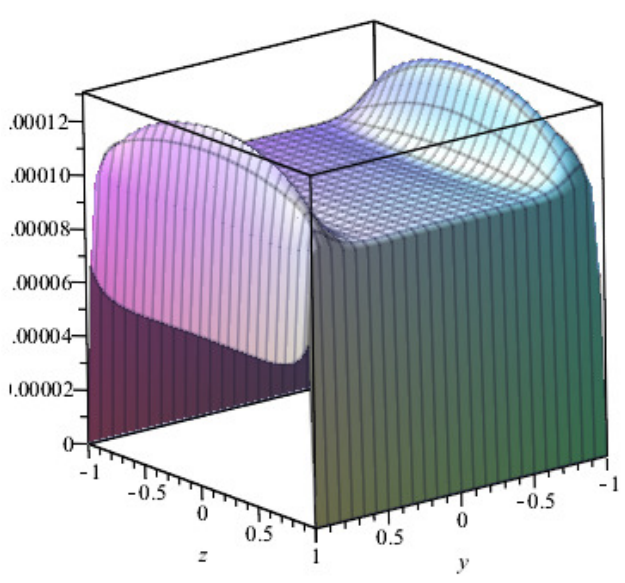

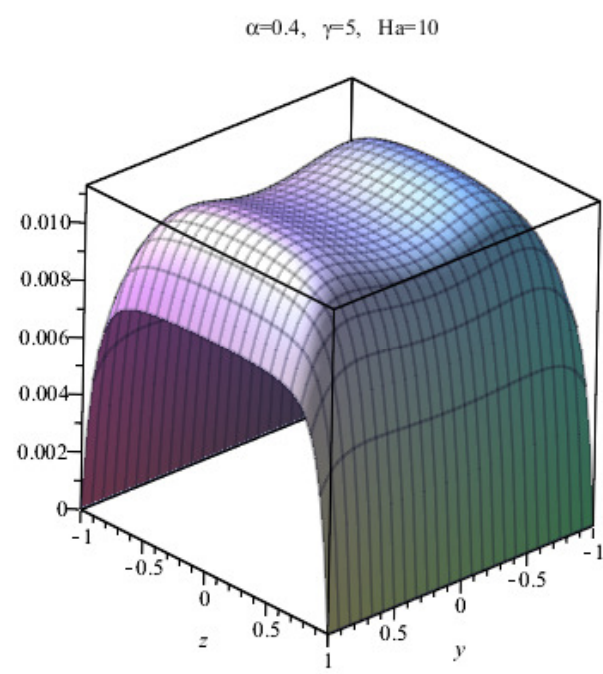

$\alpha=0.4, \quad \gamma=10, \quad \mathrm{Ha}=10$

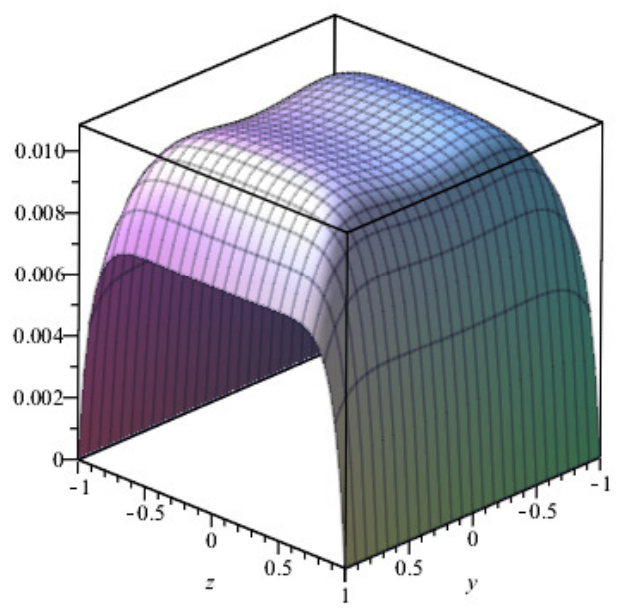

$\alpha=0.4, \gamma=10, \quad \mathrm{Ha}=100$

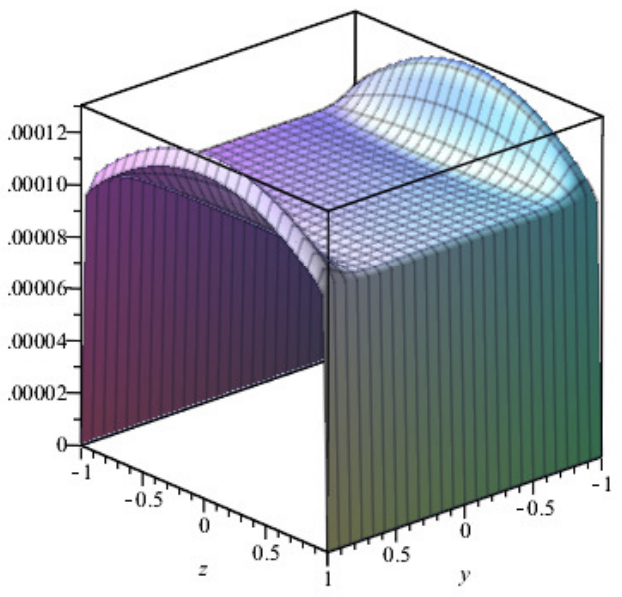

Fig. 3. Velocity distribution for $\alpha$ and $\gamma$ at $H a=10$ and $H a=100$

One can see from Fig.2, that there is a big difference between the flow in the duct for $\gamma<10$ and $\gamma>10$. For $\gamma<10$ the velocity amplitude near the side walls $y= \pm 1$ increases with the increase of the parameter $\alpha$, and the fluid velocity at the walls is much greater than the velocity in the centre. For $\gamma>10$ the flow velocity in the centre of the duct is greater than for $\gamma<10$, and the amplitude of the velocity decreases with the increase of the slip parameter $\alpha$. Besides, the flow velocity at the side walls increases with the increase of $\alpha$. One more interesting result can be seen in Fig. 2 and Fig. 3: for 
$\gamma>10$ the increase of $\gamma$ does not have essential influence on the flow velocity. Moreover, the difference between the velocity in the centre of the duct and near the side walls is not so significant as it is for $\gamma<10$. At $\gamma=50$ and $\alpha=0.4$ the velocity profile has the form of a straight line, i.e. the fluid flows with almost the same velocity along the whole section $z=0$. As it can be seen from Fig. 3, at $\alpha=0.04$ the velocity profile at the side walls $y= \pm 1$ has the M-shaped profile and that is a new and interesting result.

\section{Conclusions}

1. Analytical solution has been obtained for a new problem on MHD flow in a rectangular duct with the perfectly conducting Hartmann walls and the side walls of finite conductivity. The slip boundary conditions for velocity were applied to the channel side walls.

2. On the base of the obtained analytical solution, the velocity field has been studied numerically. The graphical representation shows a big difference in the fluid flow behavior for conductivity of the walls $\gamma<10$ and $\gamma>10$, but for all $\gamma$ values the velocity at the walls increases with the increase of the $\alpha$ value.

3. The obtained results are very interesting and requiring an additional analytical investigation, which will be made by the authors in the nearest future.

\section{References}

1. Hron J., Le Roux C., Malek J., Rajagopal K.R. Flows of incompressible fluids subject to Navier's slip on the boundary. Comput. Math. Appl., vol. 56, 2008, pp. 2128-2143.

2. Smolentsev S. MHD duct flow under hydrodynamic "slip" condition". J. Theor. Comput. Fluid Dyn., 23(6), 2009, pp. 557-570.

3. Hunt J.C.R. Magnetohydrodynamic flow in rectangular ducts. J. Fluid Mech., 21, 1956, pp. 577-590. 